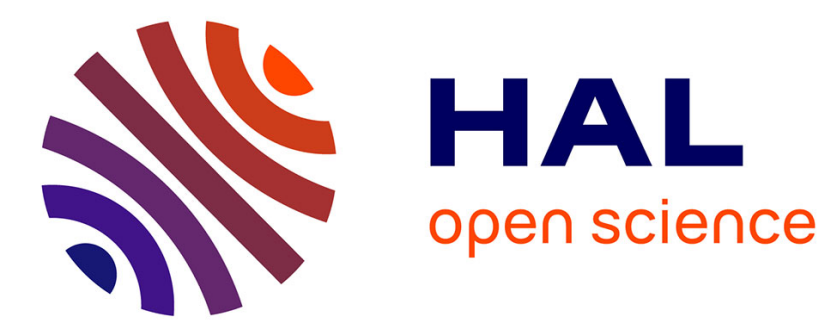

\title{
Étude des fluctuations de fréquence de lasers monomodes
}

\author{
E. Boileau, J.-M. Battifol, H. Clergeot, B. Picjnbono
}

\section{To cite this version:}

E. Boileau, J.-M. Battifol, H. Clergeot, B. Picjnbono. Étude des fluctuations de fréquence de lasers monomodes. Revue de Physique Appliquée, 1971, 6 (1), pp.23-30. 10.1051/rphysap:019710060102300 . jpa-00243499

\section{HAL Id: jpa-00243499 https://hal.science/jpa-00243499}

Submitted on 1 Jan 1971

HAL is a multi-disciplinary open access archive for the deposit and dissemination of scientific research documents, whether they are published or not. The documents may come from teaching and research institutions in France or abroad, or from public or private research centers.
L'archive ouverte pluridisciplinaire HAL, est destinée au dépôt et à la diffusion de documents scientifiques de niveau recherche, publiés ou non, émanant des établissements d'enseignement et de recherche français ou étrangers, des laboratoires publics ou privés. 


\title{
ÉTUDE DES FLUCTUATIONS DE FRÉQUENCE DE LASERS MONOMODES (*)
}

\author{
par E. BoileaU, J.-M. BATtifol, H. CleRgeOT et B. PICJNBONO \\ Institut d'Electronique Fondamentale (**), Faculté des Sciences, 91, Orsay \\ (Reçu le 15 octobre 1970)
}

\begin{abstract}
Résumé. - La largeur de raie d'un oscillateur laser monomode est essentiellement due aux fluctuations de fréquence. On admet généralement que celles-ci peuvent se décomposer en fluctuations à court terme et dérives lentes. Cependant, cette séparation n'est pas toujours justifiée, et nous avons mis au point un dispositif d'analyse permettant d'aborder ce problème. Pour ceci le signal de battement de deux lasers libres et indépendants, est transformé en impulsions qui sont analysées à l'aide d'un sélecteur multicanaux. Les résultats sont ensuite, soit visualisés sous forme d'échantillonnage de la fréquence instantanée, soit traités numériquement.

Nous étudions les fluctuations obtenues avec deux lasers de série pendant un temps d'observation $T$ variant approximativement de $10 \mathrm{~ms}$ à 1 minute. La description de ces fluctuations est faite en utilisant deux fonctions introduites antérieurement dans des études analogues, et également une nouvelle fonction qui est sur certains points plus avantageuse que les précédentes.

Les résultats montrent une possible séparation des dérives et des fluctuations à court terme. L'écart quadratique correspondant à ces dernières est d'environ $1 \mathrm{kHz}$.

Abstract. - The line-width of a monomode laser is essentially caused by frequency fluctuations. It is generally assumed that these can be separated in short term fluctuations and slow drifts. However, this separation is not always justified, and we have used a method of analysis which faces this problem. The beat signal of two free-runing independent lasers is processed to give pulses which are studied with a multichannel-analyseur. The results are then either shown on a scope as samples of the instantaneous frequency, or used for numerical computations.

We study the fluctuations obtained with serie-manufactured lasers during observation-times varying approximatively from $10 \mathrm{~ms}$ to 1 minute. The description of these fluctuations is made with two functions previously introduced in analogous studies, and also a new function which by certain aspects is more powerfull than the former. Results show a possible separation between drifts and short term fluctuations. For the latter the r. m. s. is about $1 \mathrm{kHz}$.
\end{abstract}

I. Introduction. - Depuis la découverte des sources lasers un très grand nombre de travaux théoriques et expérimentaux ont été consacrés à l'étude de leur largeur de raie ou de leur stabilité en fréquence ([1] à [8]). On sait en effet que le spectre ultime d'un oscillateur laser dû au bruit quantique de l'émission spontanée est extrêmement étroit, [9] et ne peut être mesuré directement par les méthodes de spectroscopie optiques traditionnelles. Il faut donc en général utiliser des méthodes délicates à mettre en ouvre et à analyser. Le moyen le plus simple est probablement d'étudier directement les fluctuations de fréquence de la source. En effet, pour un laser fonctionnant très au-dessus du seuil, l'amplitude du champ est bien stabilisée [10] et la largeur de raie est due pratiquement aux fluctuations de fréquence.

(*) Ce travail a été partiellement subventionné par le contrat D. R. M. E. 69-34-478.

$\left({ }^{* *}\right)$ Laboratoire associé au C. N. R. S. Adresse actuelle: Laboratoire d'étude des phénomènes aléatoires, Bâtiment 210, 91-Orsay.
Cependant, l'étude même de ces fluctuations n'est pas du tout simple. En effet, la mesure d'une grandeur statistique quelconque se fait en remplaçant des moyennes d'ensemble par des moyennes temporelles sur un temps très long, et présuppose donc une certaine stationnarité des phénomènes. Mais cette stationnarité n'existe pas pour un laser, même très bien stabilisé, et la variance des fluctuations de fréquence observée sur un temps très long (par exemple une journée) est considérable.

On est donc conduit à introduire des notions telles que celle de "spectre instantané " [11], ou pour des applications pratiques à parler de "stabilité à court terme " ou "à long terme ", qui sont des notions mal définies. En effet, sans l'expliciter complètement, on admet que les unes sont dues à des fluctuations de "fréquence instantanée " et les autres à une dérive lente de fréquence, sans savoir si ces deux classes sont nettement définies.

Le même type de problème se pose évidemment pour tous les oscillateurs, et, à notre connaissance, a été 
très peu examiné sous cet angle critique [12] à [14].

Dans le cas du laser, malgré de nombreuses mesures ces difficultés n'ont pas été réellement envisagées. Les travaux consacrés à l'étude du bruit quantique [4][6]-[7] avaient surtout pour but la vérification de formules théoriques, et dès lors utilisaient des sources fonctionnant au voisinage du seuil, ce qui élargit artificiellement beaucoup le spectre, et facilite donc les mesures. D'autres travaux [1], [2] ont utilisé des temps de mesure très courts, mais ne donnent pas des résultats généraux. Dans l'état actuel, la description statistique la plus complète, a été obtenue par Siegman et al. [5]. Ils ont étudié de trois manières différentes le signal de battement de deux lasers monomodes indépendants et identiques (Modèles Spectra Physics 119) asservis par un contrôle automatique de fréquence (C. A. F.) compensant leurs dérives relatives. Les résultats obtenus étaient qualitativement concordants, mais avec cependant certaines différences inexpliquées. De plus le choix de la constante de temps du C. A. F. présuppose que l'on sait séparer sans ambiguïté fluctuations et dérives.

C'est cette question que l'on se propose d'examiner dans ce travail. Après une courte analyse théorique du problème, nous décrivons le dispositif expérimental réalisé, ainsi que les résultats obtenus.

II. Analyse théorique des mesures de fluctuations de fréquence. - Pour présenter cette analyse il faut tout d'abord se donner un modèle statistique décrivant les fluctuations de fréquence. Les résultats dépendent évidemment de ce modèle, mais notre but n'étant pas de comparer différents modèles, nous choisissons le plus commode. Celui-ci a été introduit par Malakhov [15] dans le cas des oscillateurs, et repris fréquemment pour le laser [5], [16], [17]. Dans ce modèle on suppose que le champ optique a une amplitude constante et une phase gaussienne, de sorte que son signal analytique s'écrit

$$
Z(t)=\exp 2 \pi i\left[v_{0} t+\Phi(t)+\varphi_{0}\right]
$$

où $v_{0}$ est la fréquence moyenne du champ, $\varphi_{0}$, une variable aléatoire uniformément répartie entre 0 et 1 pour assurer la stationnarité. La phase instantanée $2 \pi \Phi(t)$ s'écrit

$$
\Phi(t)=\int_{0}^{t} \mathrm{~d} \Phi(\theta)
$$

avec

$$
\mathrm{d} \Phi(\theta)=\alpha F(\theta) \mathrm{d} \theta+\beta \mathrm{d} \psi(\theta) .
$$

Le modèle consiste à supposer que $F(t)$ est une fonction aléatoire gaussienne, stationnaire et centrée, et par conséquent complètement déterminée par sa fonction de corrélation $\Gamma_{\mathrm{F}}(\tau)$ ou par sa densité spectrale $\gamma_{\mathrm{F}}(v)$ (transformée de Fourier de $\Gamma_{\mathrm{F}}$ ). Nous désignerons par $t_{\mathrm{c}}$ le temps de corrélation de $F(t)$ et par $\sigma_{\mathrm{F}}^{2}$ son écart quadratique.

Le dernier terme de l'éq. (3) décrit le bruit quantique caractérisé par des accroissements $\mathrm{d} \psi(\theta)$ indépendants.
Le processus $\psi(t)$ est alors un mouvement brownien, défini par une constante de diffusion $\gamma$ et tel que

$$
\sigma_{\psi}^{2}=\gamma t
$$

Cependant, pour un laser fonctionnant à un niveau habituel, le bruit quantique est inobservable, par rapport aux autres fluctuations [2] et par conséquent nous poursuivons notre étude en supposant $\alpha=1$ et $\beta=0$. Indépendamment de l'avantage de commodité de ce modèle pour les calculs, remarquons qu'il est $a$ priori vraisemblable dans la mesure où les autres fluctuations proviennent essentiellement du fait que la longueur de la cavité n'est pas rigoureusement constante; or, si on admet que ces fluctuations de longueur sont gaussiennes, on peut montrer [18] que celles de $F(t)$ le sont quasiment aussi. On retrouve ici une situation bien connue en théorie du signal. Les signaux gaussiens sont pratiquement les seuls pour lesquels des calculs développés peuvent s'effectuer, et fort heureusement ils sont très fréquents en raison des théorèmes asymptotiques du calcul des probabilités (tendance vers la loi de Gauss dans la loi des grands nombres).

Comme $F(t)$ est gaussienne, on obtient aisément : [17] la fonction de corrélation de $Z(t)$, ou fonction de cohérence d'ordre un du champ,

$$
\Gamma_{z}(\tau)=E\left[Z(t) Z^{*}(t-\tau)\right]
$$

par

$$
\Gamma_{z}(\tau)=\exp \left[i \omega_{0} \tau\right] \exp \left[-\frac{1}{2}\left(\sigma_{\Delta \varphi}^{2}\right)\right]
$$

où $\Delta \varphi$ est la différence de phase

$$
2 \pi \int_{t-\tau}^{t} F(\theta) \mathrm{d} \theta
$$

ce qui donne donc

$$
\sigma_{\Delta \varphi}^{2}=4 \pi^{2} \tau^{2} \int_{-\infty}^{+\infty}\left[\frac{\sin \pi v \tau}{\pi \nu \tau}\right]^{2} \gamma_{F}(v) \mathrm{d} \nu .
$$

Le spectre optique $\gamma_{z}(v)$ s'obtient par transformation de Fourier de $\Gamma_{z}(\tau)$ et se déduit donc de $\gamma_{\mathrm{F}}(v)$. Il en est de même pour le temps de cohérence $\tau_{\mathrm{c}}$, qui est le temps de corrélation de $Z(t)$.

L'éq. (6) a deux formes asymptotiques qu'il est important de noter, selon les valeurs de $\tau$ par rapport au temps de corrélation $t_{\mathrm{c}}$ de $F(t)$. Ces formes s'écrivent

$$
\begin{array}{lll}
\sigma_{\Delta \varphi}^{2} \approx 4 \pi^{2} \sigma_{\mathrm{F}}^{2} \tau^{2} & \text { pour } \tau \ll t_{\mathrm{c}}, \\
\sigma_{\Delta \varphi}^{2} \approx 4 \pi^{2} \gamma_{\mathrm{F}}(0) \tau & \text { pour } \tau \gg t_{\mathrm{c}} .
\end{array}
$$

De plus, les résultats se simplifient beaucoup dans deux cas extrêmes [15], [18] :

a) $t_{\mathrm{c}} \sigma_{\mathrm{F}} \ll 1$, ou fréquence instantanée à corrélation microscopique.

On montre alors que le spectre optique $\gamma_{z}(v)$ est de forme lorentzienne, de largeur de raie $t_{\mathrm{c}} \sigma_{\mathrm{F}}^{2}$, et on a donc alors les inégalités

$$
t_{\mathrm{c}} \ll \frac{1}{\sigma_{\mathrm{F}}} \ll \tau_{\mathrm{c}} \approx \frac{1}{t_{\mathrm{c}} \sigma_{\mathrm{F}}^{2}} .
$$


Il se produit un phénomène de rétrécissement de la raie, puisque $t_{\mathrm{c}} \sigma_{\mathrm{F}}^{2} \ll \sigma_{\mathrm{F}}$. Ainsi dans ce cas la largeur de raie n'est pas déterminée par la variance $\sigma_{\mathrm{F}}$ des fluctuations de fréquence :

b) $t_{\mathrm{c}} \sigma_{\mathrm{F}} \gg 1$, ou corrélation à lorgue portée.

On montre alors que le spectre optique est gaussien, et proportionnel à la densité de probabilité de $F(t)$. La largeur de raie est donc $\sigma_{\mathrm{F}}$ et on a l'inégalité

$$
\tau_{\mathrm{c}} \approx \frac{1}{\sigma_{\mathrm{F}}} \ll t_{\mathrm{c}} .
$$

Cette brève discussion nous permet alors de comprendre et de commenter les résultats obtenus par Siegmann et al. [5].

Aucun détecteur actuellement disponible n'étant sensible à la phase du champ, l'étude se fait sur le battement de deux lasers indépendants et identiques. Le signal de battement a la même forme que $Z(t)$, en remplaçant $v_{0}$ par $\left|v_{1}-v_{2}\right|$, où $v_{1}$ et $v_{2}$ sont les fréquences moyennes des deux lasers, la phase $\Phi(t)$ étant alors la différence de phase entre eux.

Une première série d'expériences a montré que le spectre du signal de battement était gaussien. Ceci conduit donc à adopter l'hypothèse $b$ de corrélation longue, et en supposant les deux lasers identiques, la largeur de chacun trouvée expérimentalement était de

$$
\sigma_{\mathrm{F}} \approx \frac{1}{\tau_{\mathrm{c}}}=5,6 \mathrm{kHz} .
$$

Une seconde série analysait le «phase jitter", i. e. $\sigma_{\Delta \varphi}^{2}$. D'après l'éq. (7), pour $\tau$ assez petit, $\sigma_{\Delta \varphi}^{2}$ est proportionnel à $\tau^{2}$, la constante fournissant une valeur de $\sigma_{\mathrm{F}}^{2}$. La proportionnalité a été correctement obtenue, mais le résultat numérique donnait alors

$$
\sigma_{\mathrm{F}}^{\prime} \approx 11 \mathrm{kHz},
$$

valeur sensiblement différente de la précédente. Cette différence est restée inexpliquée. De plus, l'examen des courbes montre que $\sigma_{\Delta \varphi}^{2}$ n'est plus proportionnel à $\tau^{2}$ à partir de $10^{-6} \mathrm{~s}$. On peut donc d'après l'éq. (7) évaluer que $t_{\mathrm{c}}$ est de l'ordre de $10^{-5}$ à $10^{-3} \mathrm{~s}$. Mais alors l'inégalité $t_{\mathrm{c}} \sigma_{\mathrm{F}} \gg 1$ n'est pas réalisée.

Enfin, une troisième méthode qui sera la seule à être utilisée dans d'autres publications concernant le bruit quantique [5] et [6], étudie directement le spectre de la fréquence instantanée $F(t)$ obtenue à partir d'un discriminateur de phase. Une large part de ce spectre montrant une décroissance en $1 / v^{2}$, il était logique d'admettre un spectre total de forme lorentzienne. Mais pour en déduire sa largeur il faut connaître exactement les fréquences où cesse la courbe en $1 / v^{2}$. Ces fréquences étant celles où le dispositif cesse de fonctionner, les auteurs ont ait une estimation assez hasardeuse et obtenu la valeur

$$
\sigma_{\mathrm{F}} \approx 6,2 \mathrm{kHz},
$$

du même ordre de grandeur que la première.
Devant ces résultats il était donc intéressant de reprendre tout simplement les mesures. Mais comme indiqué ci-dessous il était surtout fondamental de savoir si les grandeurs mesurées avaient un sens, ou dépendaient de manière critique de la constante de temps $T$ du C. A. F. asservissant les deux lasers. En effet, si le $\sigma_{\mathrm{F}}$ mesuré est une fonction $\sigma_{\mathrm{F}}(T)$ toujours croissante jusqu'à $\sigma_{\mathrm{F}}(\infty)$, le concept de fluctuations de fréquence à "court terme » n'a guère de signification. Au contraire, si la densité spectrale de $F(t)$ est de la forme

$$
\gamma_{\mathrm{F}}(v)=\sigma_{1}^{2} \gamma_{1}(v)+\sigma_{2}^{2} \gamma_{2}(v)
$$

où $\gamma_{1}(v)$ et $\gamma_{2}(v)$ sont centrées sur $v=0$ et de largueurs très différentes $\left(\Delta v_{1} \ll \Delta v_{2}\right)$, il devient possible de séparer fluctuations et dérives. En effet, si on admet

$$
\int \gamma_{1}(v) d v=\int \gamma_{2}(v) d v,
$$

on voit facilement que pour $T$ nettement plus grand que $\frac{1}{\Delta v_{2}}$, la contribution de $\gamma_{2}(v)$ au $\sigma_{\mathrm{F}}$ observé est négligeable (voir formules de l'appendice B). Pourvu que cette condition soit remplie avec $T$ plus petit que $1 / \Delta v_{1}$, on $\mathrm{a} \sigma_{\mathrm{F}} \simeq \sigma_{1}$, et ceci est obtenu dans un intervalle de variation de $T$ notable; il s'agit alors de fluctuations à court terme. Evidemment, pour $T \rightarrow \infty$, le $\sigma_{\mathrm{F}}^{2}$ observé tends vers $\sigma_{1}^{2}+\sigma_{2}^{2}$.

Ceci nous conduit donc à mettre en œuvre une méthode expérimentale où $T$ apparaît nettement.

III. Description de la méthode expérimentale. L'expérience est décrite schématiquement sur la figure 1. Nous utilisons le même montage optique que

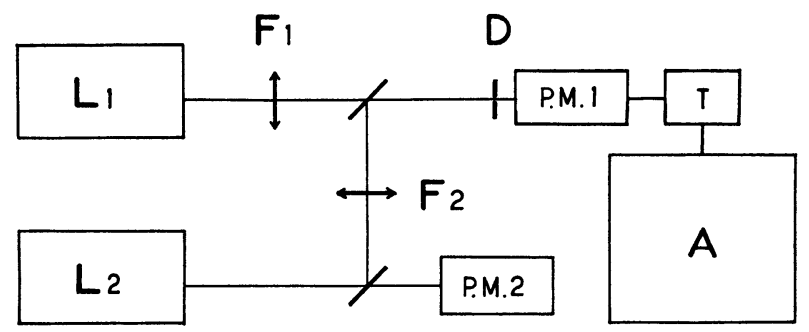

Fig. 1. - Principe de l'expérience. Le battement recueilli sur le P. M. 1 est analysé par le trigger et le sélecteur multicanaux A.

Le P. M. 2 permet de contrôler le niveau du laser 2.

celui de Siegman, consistant à faire battre deux lasers monomodes $L_{1}$ et $\mathrm{L}_{2}$ (lasers de la même fabrication : Spectra Physics 119). Cependant, ceux-ci ne sont pas asservis en fréquence, et les dérives lentes de l'un par rapport à l'autre ne sont pas corrigées. Elles le seront dans l'analyse statistique des résultats de mesure. La fréquence $\mathrm{du}$ battement dérive donc lentement au cours des mesures. Pour chaque laser, la longueur de la cavité peut être réglée par l'intermédiaire d'un cristal piézo-électrique ; ceci permet d'imposer au début de chaque mesure une fréquence du signal de battement de l'ordre de $1 \mathrm{MHz}$. Ce signal, fourni par le photomultiplicateur $\mathrm{PM}_{1}$, est transformé en une suite 
d'impulsions identiques $P_{1}$ à l'aide du trigger $T$. Ces impulsions apparaissent à chaque passage à zéro dans un sens déterminé du signal. L'intervalle entre deux impulsions $\mathbf{P}_{\mathbf{1}}$ définit donc une «période instantanée » du battement. Les impulsions sont analysées par un sélecteur 800 canaux (Intertechnique Didac 800) fonctionnant en "multiéchelle », c'est-à-dire que chaque canal fournit le nombre d'impulsions arrivées pendant une durée $\tau$ (le fonctionnement du sélecteur suppose la fréquence du battement inférieure à $10 \mathrm{MHz}$ ).

Les comptages se font à intervalles périodiques de période $T_{i}$. L'usage le plus simple consiste à réaliser $\tau=T_{i}$, à un temps mort près (inférieur à $5 \mu \mathrm{s}$ ). Mais, pour l'analyse statistique, il est nécessaire de faire des mesures avec $\tau<T_{i}$, comme ceci apparaît sur la figure $2 a$. Dans ce cas, les impulsions $\mathrm{P}_{2}$ de période $T_{i}$ fournies par l'analyseur servent à déclencher un générateur fournissant des impulsions $\mathbf{P}_{3}$ de durée $\tau$. Un système de blocage permet de ne compter que les impulsions parvenues pendant la durée $\tau$.

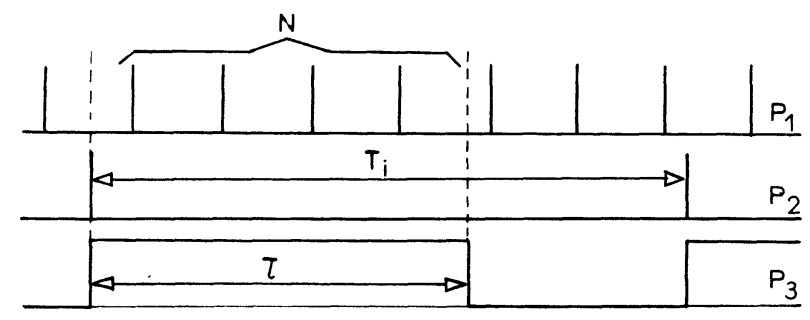

(a)

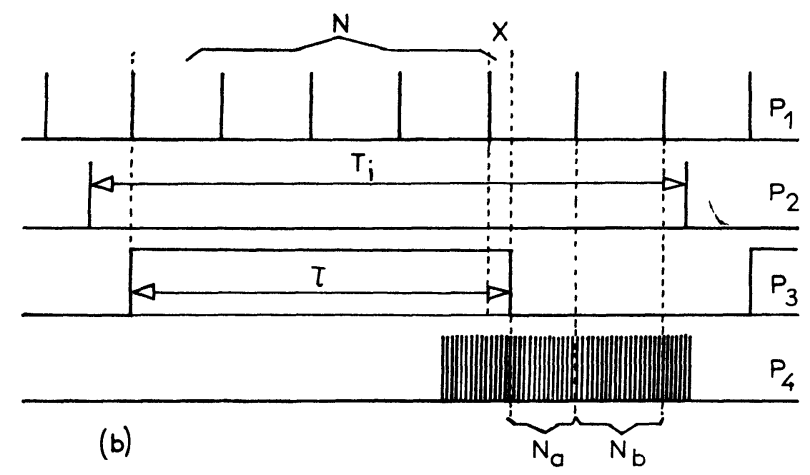

Fig. 2. - Principe de l'analyse des impulsions fournies par le trigger. (2a) Analyse avec une erreur de l'ordre de $1 / \tau ;(2 b)$. Principe d'une interpolation linéaire plus fine.

Soit $N(t, \tau)$ le nombre d'impulsions comptées dans l'intervalle $t-(\tau / 2), t+(\tau / 2)$. Ce nombre nous donne une estimation de la moyenne temporelle $g(t, \tau)$ de la fréquence instantanée $F(t)$ pendant cet intervalle, définie par

$$
g(t, \tau)=\frac{1}{\tau} \int_{t-\tau / 2}^{t+\tau / 2} F(\theta) \mathrm{d} \theta
$$

On a en effet

$$
g(t, \tau)=\frac{1}{\tau}[N(t, \tau)+X]
$$

où $|X|<1$, et il en suit que $g(t, \tau)$ est mesurée avec une erreur inférieure à $1 / \tau$.
Bien que ce ne soit pas réalisé expérimentalement dans ce travail, il est possible d'améliorer cette précision, en particulier lorsqu'on utilise de faibles valeurs de $\tau$ [19]. Le principe de la méthode est indiqué sur la figure $2 b$. Il suffit d'utiliser des impulsions $\mathbf{P}_{4}$ fournies par une horloge rapide et de compter les nombres $N, N a$ et $N b$. On voit alors simplement qu'une meilleure approximation de $g$ est fournie en utilisant une interpolation linéaire remplaçant $N$ par

$$
N+(N b-N a) / N b \text {. }
$$

Finalement, les 800 nombres fournis par le sélecteur sont stockés pour en faire l'analyse statistique décrite ci-dessous. Ils peuvent également être visualisés sur un écran en vue d'une analyse rapide.

Celle-ci est très utile car elle permet sans aucun traitement numérique de détecter les paramètres les plus importants influençant la stabilité, et ainsi d'améliorer au maximum les conditions d'emploi avant toute mesure plus fine. Ces paramètres sont d'ordre mécanique, électrique et thermique.

Il faut minimiser les vibrations mécaniques des lasers qui élargissent considérablement et artificiellelement la largeur de raie. Ainsi les sources sont montées sur des tables antivibratoires particulièrement soignées et entourées d'une protection contre les vibrations acoustiques.

Nous avons obtenu une amélioration notable de la stabilité en remplaçant l'alimentation du constructeur, servant à alimenter le cristal piézo-électrique qui supporte un des miroirs, par une alimentation plus stable.

L'instabilité thermique crée également une variation de longueur de la cavité et donc de fréquence de l'oscillateur. Nos lasers comportaient initialement une stabilisation de température par résistances chauffantes alimentées en courant $50 \mathrm{~Hz}$ redressé. Cependant, nous avons constaté [18] que la stabilité à court terme
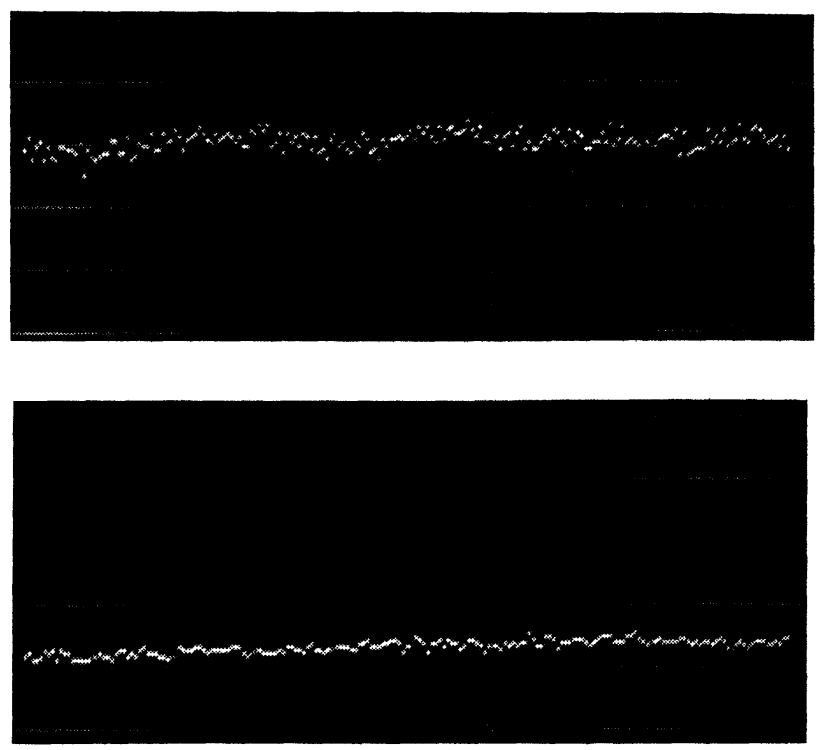

FIG. 3. - Exemple d'analyse rapide du battement. Unité verticale $20 \mathrm{kHz}$. Unités horizontales : $a .-200 \mathrm{~ms} ; b .-20 \mathrm{~ms}$. 
est nettement améliorée en supprimant complètement cette stabilisation thermique. Des exemples de tels résultats apparaissent sur les figures 3 et 4 , montrant une fluctuation à court terme et une dérive lente. Mais comme nous l'avons indiqué, ces notions méritent d'être approfondies, et ceci est l'objet du prochain paragraphe.

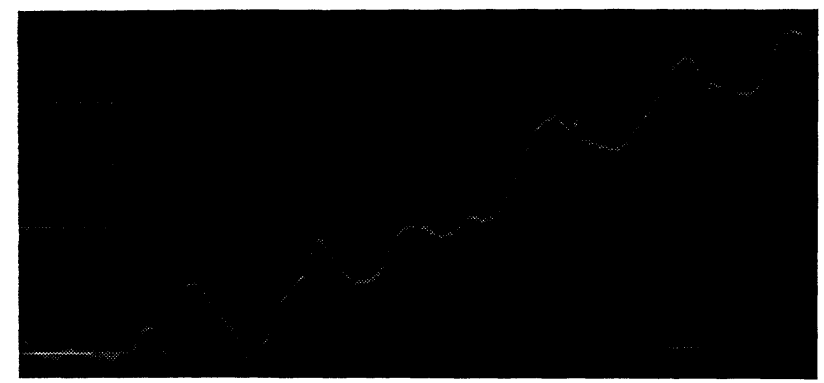

Fig. 4. - Autre exemple d'analyse rapide. Unité verticale $1 \mathrm{MHz}$; Unité horizontale : $80 \mathrm{~s}$.

IV. Analyse statistique et résultats expérimentaux. - 1. Principe de l'ANAlyse. - Comme nous l'avons précédemment montré nous obtenons expérimentalement des échantillons de la fonction $g(t, \tau)$, valeur moyenne de la fréquence sur l'intervalle de temps $\tau$. Cette constante de temps $\tau$ est tout à fait arbitraire, et nous pouvons aisément la faire varier. Il est bien clair que, même lorsque ce n'est pas spécifié, elle apparaît dans tous les dispositifs de mesure de fréquence qui font nécessairement une moyenne temporelle. Dans les dispositifs utilisant un C. A. F. il apparaît une autre constante de temps $T$, en général mal définie et pouvant très peu varier. C'est pourquoi nous avons supprimé tout $C$. A. F. et utilisé une méthode d'élimination des dérives où $T$ est parfaitement défini et susceptible de varier à volonté.

Pour ceci il suffit de traiter directement la fonction $g(t, \tau)$. Diverses méthodes sont alors possibles.

Une première possibilité mentionnée par Barnes [13] consiste à introduire la variation

$$
\Delta g(t, T, \tau)=g\left(t+\frac{T}{2}, \tau\right)-g\left(t-\frac{T}{2}, \tau\right)
$$

décrivant l'accroissement de $g$ pendant l'intervalle $T$ qui élimine donc les dérives de fréquence inférieure à $1 / T$. Comme $\Delta g$ est aléatoire, on peut se faire une idée de ses fluctuations en déterminant sa variance $\sigma_{\Delta g}$.

Une seconde possibilité mentionnée par d'autres auteurs [12], [14] consiste à introduire un écart quadratique temporel $U$ éliminant aussi les dérives lentes et défini par

$$
\begin{aligned}
U & =\hat{\sigma}_{g}^{2}(t, T, \tau) \\
& =\frac{1}{T} \int_{t-T / 2}^{t+T / 2} g^{2}(\theta, \tau) \mathrm{d} \theta-\left[\frac{1}{T} \int_{t-T / 2}^{t+T / 2} g(\theta, \tau) \mathrm{d} \theta\right]^{2}
\end{aligned}
$$

Cette grandeur étant aléatoire, on peut utiliser sa moyenne, et poser

$$
\tilde{\sigma}_{g}^{2}(T, \tau)=E\left[\hat{\sigma}_{g}^{2}(t, T, \tau)\right]
$$

Cependant, la valeur moyenne est évidemment insuffisante pour décrire une variable aléatoire, et nous montrons en Appendice A que moyennant des hypothèses très générales, la variance de $U$ est du même ordre de grandeur que sa moyenne, de sorte qu'on peut prendre la moyenne comme indication des fluctuations de fréquence.

Pour différentes raisons [18] nous avons introduit une troisième fonction définie par

$$
d(t, T, \tau)=g(t, \tau)-g(t, T)
$$

dont l'interprétation est immédiate. Sa variance $\sigma_{d}$ est voisine de $\sigma_{\Delta g}$, mais on peut montrer que l'élimination des basses fréquences est plus efficace sur $\sigma_{d}$ que sur $\sigma_{\Delta g}$ ou $\tilde{\sigma}_{g}$. De plus l'étude de $\sigma_{d}$ en fonction de $T$ peut fournir des informations sur la partie basse fréquence de $\gamma_{\mathrm{F}}(v)$ dans des conditions moins restrictives (voir Appendice B).

Finalement nous devons noter que notre méthode expérimentale ne nous fournit pas $g(t, T)$, mais seulement des échantillons de cette fonction aux instants, $t_{n}=t+n T_{i}$. Ainsi les moyennes temporelles sont obtenues par sommes discrètes à partir d'échantillons et l'approximation ainsi introduite est étudiée dans l'Appendice $\mathrm{C}$.

2. RÉSUltatS EXPÉRIMENTAUX. - Chaque expérience donne 800 valeurs de $N(t, \tau)$ pour des valeurs imposées de $\tau$ et $T_{i}$. A l'aide de ces valeurs un ordinateur calcule $\sigma_{\Delta g}(\tau, T), \tilde{\sigma}_{g}(\tau, T)$ et $\sigma_{d}(\tau, T)$ pour des valeurs de $T$ allant de $3 T_{i}$ à $65 T_{i}$. Ces variances sont calculées pour un laser, en supposant que les deux sources contribuent également aux fluctuations du battement. Si l'on répète plusieurs fois la même expérience, les résultats obtenus ne sont évidemment pas absolument identiques, ce qui peut être dû au nombre trop petit d'échantillons traités ou à des variations au cours du temps des conditions expérimentales. C'est pourquoi on n'obtient pas en général une courbe continue pour des mesures avec des $T_{i}$ différents.

Un exemple typique de résultats apparaît sur la figure 5. Comme indiqué précédemment les lasers

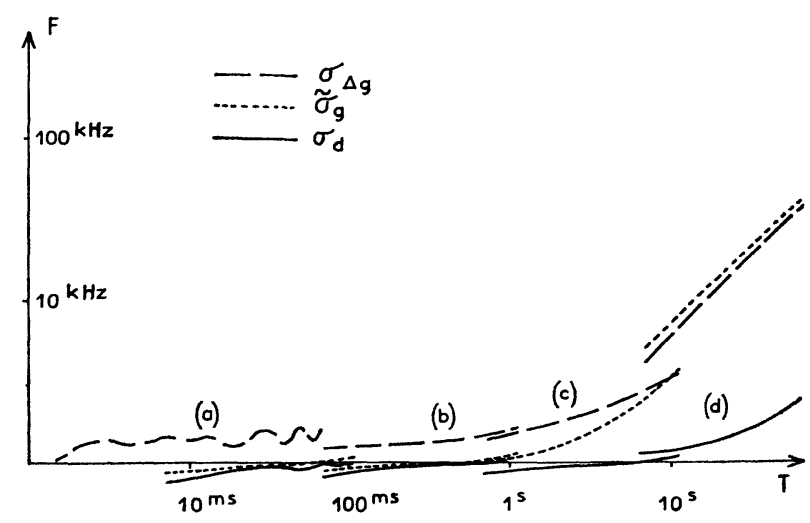

FIG. 5. - Exemple de résultat d'analyse statistique obtenu avec $\tau=1 \mathrm{~ms}$. 
fonctionnent sans régulation thermique. La constante de temps $\tau$ vaut $1 \mathrm{~ms}$ et $T_{i}$ vaut respectivement pour les $\operatorname{arcs}(a),(b),(c),(d) 1,10,100 \mathrm{~ms}$ et $1 \mathrm{~s}$.

On voit qu'entre $10 \mathrm{~ms}$ et $1 \mathrm{~s}$, les variances mesurées par les trois méthodes sont de l'ordre de $1 \mathrm{kHz}$, ce qui est une nette amélioration par rapport aux résultats de Siegman (cette amélioration est en rapport avec les précautions décrites au III). De plus, ces résultats semblent peu dépendre de $T$ pour $T<1 \mathrm{~s}$, ce qui suggère la possibilité d'une séparation entre fluctuations à court terme et dérives lentes. Cependant, les résultats sont de l'ordre de la précision du dispositif. Ils sont effectivement légèrement améliorés en prenant $\tau=10 \mathrm{~ms}$ (voir Fig. 6).

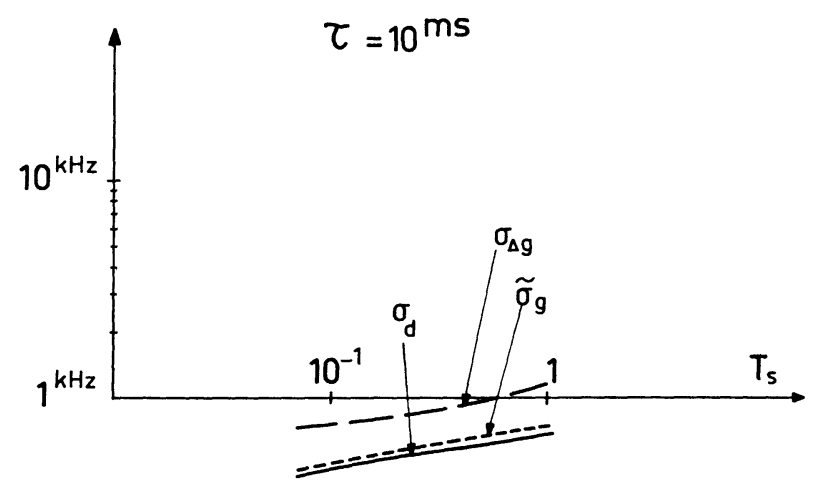

Fig. 6. - Résultat obtenu avec $\tau=10 \mathrm{~ms}$.

Par ailleurs, la pente des courbes pour $T>10 \mathrm{~s}$ est particulièrement reproductible (pente 1 en coordonnée Log-Log), et on montre en Appendice B que ceci est en bon accord avec une densité spectrale du type $\gamma_{\mathrm{F}}(v)=k /\left[\mathrm{B}^{3}+|v|^{3}\right]$ aux basses fréquences, domaine qui ne pouvait être atteint par les mesures de Siegman.

V. Conclusion. - La méthode de mesure que nous avons mise au point peut s'appliquer à l'étude de tous les oscillateurs. Aux fréquences plus basses on peut même évidemment se dispenser du battement. Elle est susceptible de nombreuses améliorations, dont certaines en cours de réalisation. La précision statistique peut s'augmenter par l'utilisation d'un sélecteur à plus 800 canaux; celle de la mesure des fréquences moyennes par le dispositif d'interpolation précédemment indiqué. Enfin on peut envisager des dispositifs plus complexes fournissant en plus de la variance la fonction de corrélation pour un temps d'analyse bien déterminé.

Appendice A. - Comparaison de $E|U|$ ET DE $\sigma_{U}$. - Le calcul de $\sigma_{U}^{2}$ nécessite d'après l'éq. (19) la connaissance des moments du quatrième ordre de $g(t$, $\tau)$. Lorsque $F(t)$ est gaussienne le résultat s'obtient comme attendu à partir de la densité spectrale $\gamma_{\mathrm{F}}(v)$. L'expression explicite s'obtient aisément par les méthodes de l'analyse harmonique [18] et l'on obtient

$$
\begin{aligned}
& \sigma_{U}^{2}(T, \tau)= \\
& =2 \int_{-}^{+} \int_{\infty}^{\infty} \gamma_{\mathrm{F}}(v) \gamma_{\mathrm{F}}\left(v^{\prime}\right)\left[\frac{\sin \pi v \tau}{\pi v \tau}\right]^{2}\left[\frac{\sin \pi v^{\prime} \tau}{\pi v^{\prime} \tau}\right]^{2} \times \\
& \quad \times\left[\frac{\sin \pi\left(v+v^{\prime}\right) T}{\pi\left(v+v^{\prime}\right) T}-\frac{\sin \pi v T}{\pi v T} \cdot \frac{\sin \pi v^{\prime} T}{\pi v^{\prime} T}\right]^{2} \mathrm{~d} v \mathrm{~d} v^{\prime}
\end{aligned}
$$

A l'aide de cette expression nous avons calculé sur ordinateur $\sigma_{U}$ pour des densités spectrales gaussienne et lorentzienne de largeur $B$. On constate dans les deux cas que si $T>1 / B$, alors $\sigma_{U} \ll E[U]$, ce qui est bien naturel en raison de la forte intégration. Dans ce cas $U$ a des fluctuations très faibles et $E[U]$ donne une bonne description de $U$. Par contre si, comme c'est le cas dans nos expériences, $T<1 / B$, $\sigma_{U}$ et $E(U)$ sont du même ordre de grandeur, et on a toujours

$$
\sigma_{U}^{2}<2 E^{2}(U) .
$$

Appendice B. - REMARQUeS SUR LES FONCTIONS $\sigma_{\Delta g}, \tilde{\sigma}_{g}$ et $\sigma_{d} \cdot-$ En utilisant les méthodes de l'analyse harmonique des fonctions aléatoires et du filtrage linéaire, ces trois variances se calculent aisément et valent

$\sigma_{\Delta g}^{2}(T, \tau)=4 \int_{-\infty}^{+\infty} \sin ^{2} \pi v T\left[\frac{\sin \pi \nu \tau}{\pi \nu \tau}\right]^{2} \gamma_{F}(v) \mathrm{d} v$

$\tilde{\sigma}_{g}^{2}(T, \tau)=\int_{-\infty}^{+\infty}\left[\frac{\sin \pi v \tau}{\pi \nu \tau}\right]^{2}\left[1-\left[\frac{\sin \pi \nu T}{\pi v T}\right]^{2}\right] \gamma_{\mathrm{F}}(v) \mathrm{d} \nu$,

$\sigma_{d}^{2}(T, \tau)=\int_{-\infty}^{+\infty}\left[\frac{\sin \pi v \tau}{\pi v \tau}-\frac{\sin \pi v T}{\pi v T}\right]^{2} \gamma_{\mathrm{F}}(v) \mathrm{d} v$

Ces expressions sont tout à fait similaires et se présentent comme intégrale de produits $\gamma_{\mathrm{F}}(v) P(T, \tau, v)$, $P$ étant un facteur de pondération éliminant pratiquement les fréquences telles que $v<1 / T$ (dérives très lentes) et $v>1 / \tau$ (fluctuations trop rapides).

Cependant pour $v \rightarrow 0, \quad P_{d} \simeq \alpha v^{4}$, tandis que $P_{\Delta g}$ ou $P_{g}$ sont du type $\alpha v^{2}$. Ceci indique que l'élimination de la dérive est plus effective par $P_{d}$ que par $P_{\Delta g}$ ou $P_{g}$. Il en résulte que l'étude de $\sigma_{d}$ peut, dans certains cas, donner des informations sur $\gamma_{F}(v)$ que l'étude de $\sigma_{\Delta g}$ ou $\tilde{\sigma}_{g}$ ne peut donner.

Ainsi, si on suppose.que

$$
\gamma_{\mathrm{F}}(v) \sim \frac{1}{|v|^{\alpha}} \text { pour } v \rightarrow 0
$$

avec $3<\alpha<5$, on obtient des intégrales divergentes pour $\sigma_{\Delta g}$ et $\tilde{\sigma}_{g}$ alors que $\sigma_{d}$ converge. Il y a lieu de remarquer qu'en réalité, le nombre de mesures étant toujours fini, chaque mesure ayant une durée finie, le traitement des résultats donne toujours des quantités bornées. Autrement dit, pratiquement, l'élimination des très basses fréquences est très énergique et il paraît présomptueux de chercher à obtenir le compor- 
tement de $\gamma_{\mathrm{F}}(v)$ pour $v \rightarrow 0$. C'est pourquoi nous avons utilisé, au lieu du spectre $\gamma_{\mathrm{F}}(v)=\frac{1}{|v|} \alpha$ (flicher noise) la forme

$$
\gamma_{\mathbf{F}}(v)=\frac{B^{(\alpha-1)}}{B^{\alpha}+|v|^{\alpha}}
$$

avec laquelle on n'a plus d'intégrales divergentes.

En utilisant (B.5) nous avons tracé des courbes représentant les fonctions $\sigma$, les intégrales étant calculées sur ordinateur. On remarque que ces fonctions de $T$, $\tau$ et $B$ ne dépendent en réalité que de deux variables; par exemple, avec

$$
M=T / \tau \quad \text { et } \quad A=B \tau,
$$

on a

$$
\begin{aligned}
\sigma_{d}(T, \tau) & =\int \frac{B^{\alpha-1}}{B^{\alpha}+|v|^{\alpha}}\left[\frac{\sin \pi \nu \tau}{\pi \nu \tau}-\frac{\sin \pi v \tau}{\pi v \tau}\right]^{2} \mathrm{~d} v \\
& =\int \frac{A^{\alpha-1}}{A^{\alpha}+|v|^{\alpha}}\left[\frac{\sin \pi x}{\pi x}-\frac{\sin \pi M x}{\pi M x}\right]^{2} \mathrm{~d} x
\end{aligned}
$$

La figure 7 donne à titre d'exemple les courbes obtenues pour $B \tau=10^{-4}$ avec $\alpha=3$, en coordonnées $(\log T / \tau, \log \sigma)$.

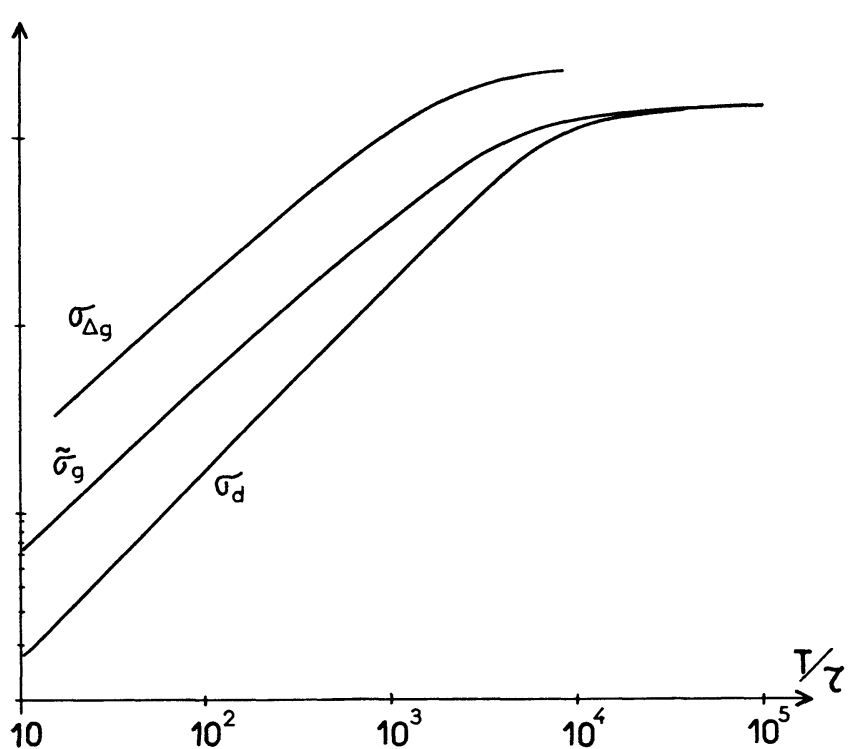

FIG. 7. - Courbes obtenues avec

$\gamma_{F}(v)=\frac{B^{2}}{B^{3}+|v|^{3}}$ et $\quad B \tau=10^{-4}$.

Avec $2<\alpha<3$, on obtient des courbes qui pour $T \ll 1 / B$, sont analogues aux droites correspondant au flicher noise [14]. Ceci provient du fait que la contribution de l'intervalle $|v|<1 / T$ est relativement faible dans les intégrales, et des approximations grossières montrent que

$$
\sigma \sim\left[\frac{T}{\tau}\right]^{\frac{\alpha-1}{2}}
$$

Lorsque $\alpha$ augmente, l'importance relative de cet intervalle augmente ; sa contribution est en $T / \tau$ pour $\sigma_{\Delta g}$ et $\tilde{\sigma}_{g}$ alors qu'elle est en $\left[\frac{T}{\tau}\right]^{2}$ pour $\sigma_{d}$. On constate que cette contribution est prépondérante dans le premier cas dès que $\alpha>3$, et la pente obtenue est 1 , indépendamment de la valeur de $\alpha$; au contraire, pour $\sigma_{d}$, cette pente augmente encore pour $3<\alpha<5$ sensiblement comme l'indique (B.7), puis est approximativement constante et égale à 2 pour $\alpha>5$.

La figure 8 a été obtenue en utilisant, au lieu de (B.5) un spectre gaussien :

$$
\gamma_{\mathrm{F}}(v)=\frac{1}{B \sqrt{2 \pi}} \exp \left[-\frac{v^{2}}{2 B^{2}}\right] .
$$

Les pentes obtenues sont les limites précédemment signalées : 1 pour $\sigma_{\Delta g}$ et $\tilde{\sigma}_{g}$, et 2 pour $\sigma_{d}$.

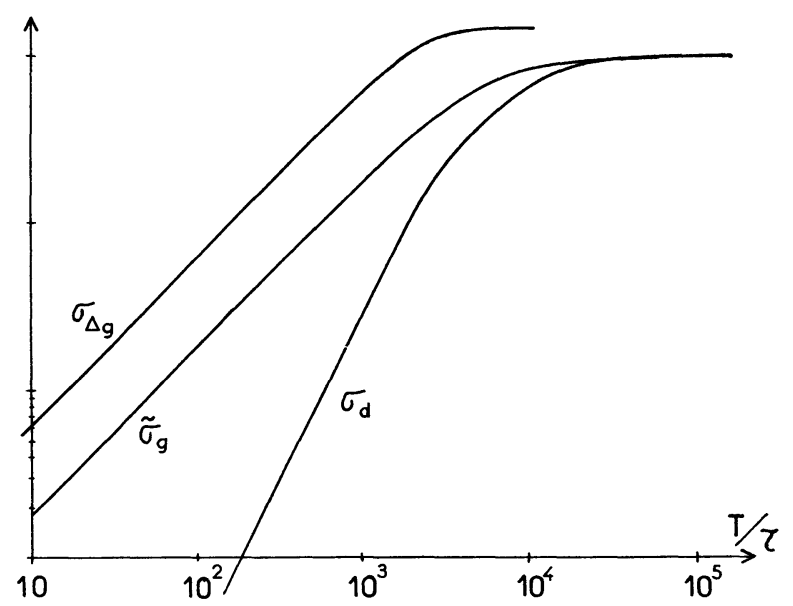

FIG. 8. - Courbes obtenues avec $\gamma_{F}(v)$ gaussienne et $B \tau=10^{-4}$.

Appendice C. - ApProximations faites dans LE CALCUL DE $U(t, T, \tau)$ ET $d(t, T, \tau)$. - L'évaluation de $d(t, T, \tau)$ définie par (21) nécessite deux mesures simultanées avec des durées respectives $\tau$ et $T$. Le calcul de $U$ défini par (19) nécessite la connaissance, pour tout $t$, de la fonction $g^{2}(t, T)$. Nos mesures ne donnent qu'un échantillonnage à des instants discrets de la fonction $g(t, \tau)$; aussi nous approximons les intégrales par des sommes. Par exemple avec $T=n T_{i}=(2 q+1) T_{i}$ nous prenons

$$
\begin{aligned}
g(t, T) & =\frac{1}{T} \int_{t-T / 2}^{t+T / 2} g(\theta, t) \mathrm{d} \theta \\
& \simeq \frac{1}{n_{k}} \sum_{=-q}^{q} g\left(t+k T_{i}, \tau\right) .
\end{aligned}
$$

Nous obtenons ainsi une fonction $d^{\prime}(t, T, \tau)$ au lieu de $d(t, T, \tau)$ et la variance correspondante est

$\sigma_{d^{\prime}}^{2}\left(n T_{i}, \tau\right)=\int\left[\frac{\sin \pi v \tau}{\pi v \tau}\right]^{2}\left[1-\frac{\sin n \pi v T_{i}}{n \sin \pi v T_{i}}\right]^{2} \gamma_{\mathrm{F}}(v) \mathrm{d} v$

Avec les approximations similaires nous obtenons une fonction $U^{\prime}$ au lieu de $U$ et sa moyenne est 
$\tilde{\sigma}_{g^{\prime}}^{2}\left(n T_{i}, \tau\right)=\int\left[\frac{\sin \pi v \tau}{\pi v \tau}\right]^{2}\left[1-\left[\frac{\sin n \pi v T_{i}}{n}\right]^{2}\right] \gamma_{\mathrm{F}}(v) \mathrm{d} v$. Nous voyons que les composantes de $\gamma_{\mathrm{F}}(v)$ corres(C.3) minées dans (C.2) et (C.3).

\section{Bibliographie}

[1] JAVAn (A.), Ballik (E. A.) and Bond (W. L.), J. [10] Freed (C.) and Haus (H. A.), I. E. E. E. J. of Q. E., Opt. Soc. Am., 1962, 52, 96.

[2] JASEJA (T. S.), JAVAN (A.) and Townes (C. H.), Phys. Rev. Letters, 1963, 10, 165.

[3] Boersh (H.) and Herziger (G.), I. E. E. E. J. of Q. E., $1966,2,549$.

[4] Zaitsev (Yu. N.) and Stepanov (D. P.), J. E. T. P. Letters, 1967, 6, 209.

[5] Siegman (A. E.), Daino (B.) and Manes (K. R.), I. E. E. E. J. of Q. E., 1967, 3, 180.

[6] Sibgman (A. E.) and Arrathoon (R.), Phys. Rev. Letters, 1968, 20, 901.

[7] Arrathoon (R.) and Siegman (A. E.), J. of Appl. Phys., 1969, 40, 910.

[8] Freed (C.), I. E. E. E. J. of Q. E., 1968, 4, 404.

[9] Lamb (W. E.), Phys. Rev., 1964, 134, 1429.

1966, 2, 190.
[11] Blanc-Lapierre (A.) et Picinbono (B.), Publ. Scient. de l'Univ. d'Alger, 1955.

[12] Cutler (L. S.) and Searle (C. L.), Proc. of the I. E. E. E. ; 1966, 54, 136 .

[13] Barnes (J. A.), Proc. of the I. E. E. E., 1966, 54, 207.

[14] Allan (D. W.), Proc. of the I. E. E. E., 1966, 54, 221.

[15] Malakhov (A. M.), J. E. T. P., 1956, 653.

[16] LAMB (W. E.), ( Quantum Optics and Electronics ), Ed. Cde Witt et al. Gordon Breach Sc. Publisher Inc. N. Y., 1965.

[17] Picinbono (B.) et Boileau (E.), J. of Soc. Am., 1968, 58, 784.

[18] BolleaU (E.), Thèse Fac. des Sciences d'Orsay, 1970.

[19] Boileau (E.) et Picinbono (B.), C. R. Acad. Sci. Paris, 1967, 255, 1408. 